\title{
Independent evolution of tetraloop in enterovirus oriL replicative element and its putative binding partners in virus protein 3C
}

\author{
Maria A Prostova ${ }^{\text {Corresp.. }}{ }^{1}$, Andrei A Deviatkin ${ }^{1}$, Irina 0 Tcelykh ${ }^{1,2}$ ， Alexander N Lukashev ${ }^{1,3}$, Anatoly P Gmyl ${ }^{1,}$ \\ 2,3 \\ ${ }^{1}$ Chumakov Institute of Poliomyelitis and Viral Encephalitides, Moscow, Russia \\ 2 Lomonosov Moscow State University, Moscow, Russia \\ 3 Sechenov First Moscow State Medical University, Moscow, Russia \\ Corresponding Author: Maria A Prostova \\ Email address: prostova_ma@chumakovs.su
}

Background. Enteroviruses are small non-enveloped viruses with (+) ssRNA genome with one open reading frame. Enterovirus protein $3 C$ (or $3 C D$ for some species) binds the replicative element oriL to initiate replication. The replication of enteroviruses features low fidelity, which allows the virus to adapt to the changing environment on the one hand, and requires additional mechanisms to maintain the genome stability on the other. Structural disturbances in the apical region of oriL domain $d$ can be compensated by amino acid substitutions in positions 154 or 156 of 3C (amino acid numeration corresponds to poliovirus $3 \mathrm{C}$ ), thus suggesting the co-evolution of these interacting sequences in nature. The aim of this work was to understand co-evolution patterns of two interacting replication machinery elements in enteroviruses, the apical region of oriL domain $d$ and its putative binding partners in the $3 \mathrm{C}$ protein.

Methods.To evaluate the variability of the domain d loop sequence we retrieved all available full enterovirus sequences (>6400 nucleotides), which were present in the NCBI database on February 2017 and analysed the variety and abundance of sequences in domain $d$ of the replicative element oriL and in the protein 3C.

Results. A total of 2,842 full genome sequences was analysed. The majority of domain d apical loops were tetraloops, which belonged to consensus $Y N H G(Y=U / C, N=$ any nucleotide, $H=A / C / U)$. The putative RNA-binding tripeptide 154-156 (Enterovirus C 3C protein numeration) was less diverse than the apical domain d loop region and, in contrast to it, was species-specific.

Discussion. Despite the suggestion that the RNA-binding tripeptide interacts with the apical region of domain $d$, they evolve independently in nature. Together, our data indicate the plastic evolution of both interplayers of 3C-oriL recognition. 
2 replicative element and its putative binding partners in

3 virus protein $3 \mathrm{C}$

4 Maria A. Prostova ${ }^{1}$, Andrei A. Deviatkin $^{1}$, Irina O. Tcelykh ${ }^{1,2}$, Alexander N. Lukashev ${ }^{1,3}$,

5 Anatoly P. Gmyl11,2,3

6 1 - Chumakov Institute of Poliomyelitis and Viral Encephalitides, Moscow, Russian

Federation

2 - Lomonosov Moscow State University, Moscow, Russian Federation

Corresponding author:

Email address:

14 prostovna@gmail.com 

with one open reading frame. Enterovirus protein $3 \mathrm{C}$ (or 3CD for some species) binds the replicative element oriL to initiate replication. The replication of enteroviruses features low

20 fidelity, which allows the virus to adapt to the changing environment on the one hand, and

21 requires additional mechanisms to maintain the genome stability on the other. Structural

22 disturbances in the apical region of oriL domain $d$ can be compensated by amino acid

23 substitutions in positions 154 or 156 of $3 \mathrm{C}$ (amino acid numeration corresponds to poliovirus

$243 \mathrm{C}$ ), thus suggesting the co-evolution of these interacting sequences in nature. The aim of this

25 work was to understand co-evolution patterns of two interacting replication machinery elements

26 in enteroviruses, the apical region of oriL domain $\mathrm{d}$ and its putative binding partners in the $3 \mathrm{C}$

27 protein.

Methods. To evaluate the variability of the domain d loop sequence we retrieved all

30 available full enterovirus sequences (>6400 nucleotides), which were present in the NCBI

31 database on February 2017 and analysed the variety and abundance of sequences in domain $\mathrm{d}$ of 32 the replicative element oriL and in the protein $3 \mathrm{C}$. 
35 apical loops were tetraloops, which belonged to consensus $\mathrm{YNHG}(\mathrm{Y}=\mathrm{U} / \mathrm{C}, \mathrm{N}=$ any nucleotide,

$36 \mathrm{H}=\mathrm{A} / \mathrm{C} / \mathrm{U}$ ). The putative RNA-binding tripeptide 154-156 (Enterovirus $C 3 \mathrm{C}$ protein

37 numeration) was less diverse than the apical domain d loop region and, in contrast to it, was

38 species-specific.

40 Discussion. Despite the suggestion that the RNA-binding tripeptide interacts with the apical

41 region of domain $\mathrm{d}$, they evolve independently in nature. Together, our data indicate the plastic

42 evolution of both interplayers of 3C-oriL recognition.

\section{Introduction}

47 proteins, 5' and 3' NTRs (non translated regions), and polyA on the 3' end (Palmenberg,

48 Neubauer and Skern, 2010). (Figure 1). Most non-structural enterovirus proteins are

49 polyfunctional. Protease $3 \mathrm{CD}$ is a precursor of polymerase $3 \mathrm{D}$ and plays a key role in the

50 initiation of replication (Harris et al., 1992; Gamarnik and Andino, 1998; Thompson and

51 Peersen, 2004). After translation by host cell ribosomal machinery, the genome is utilized for the

52 synthesis of the (-) strand RNA, which, in turn, serves as a matrix for the synthesis of multiple 
53 daughter $(+)$ strands. Non-translated regions of the genome and a coding sequence within the

54 genomic region encoding the viral helicase $2 \mathrm{C}$ contain replicative elements, which interact with

55 viral and host proteins. These RNA-protein complexes regulate initiation and further steps of

56 replication. For poliovirus, the most clinically relevant member of the Enterovirus genus, there

57 are at least three known RNA-protein complexes, which are formed with the replicative elements

58 oriL, oriR and oriI during replication (Figure 1).

59 Complex oriL with viral protein 3CD and the host protein PCBP2 is crucial for the

60 transcription initiation (Goodfellow et al., 2000; Vogt and Andino, 2010; Chase, Daijogo and

61 Semler, 2014). The element oriL has a cloverleaf-like secondary structure with four domains

62 termed a (stem of the cloverleaf), b, c and d (leaves of the cloverleaf) (Trono, Andino and

63 Baltimore, 1988; Andino, Rieckhof and Baltimore, 1990) (Figure 1). Previously, it was

64 demonstrated in vitro that $3 \mathrm{CD}$ (or $3 \mathrm{C}$ ) of poliovirus, coxsackievirus $\mathrm{B} 3$ and bovine enterovirus

651 interacts with the apical loop and the flanking base pairs of hairpin $d$ in the oriL element

66 (Andino, Rieckhof and Baltimore, 1990; Du et al., 2003; Ihle et al., 2005) (Figure 1). genera were shown by NMR experiments to be tetraloops with a specific spatial structure, which

69 belongs to the UNCG structural class of stable tetraloops (Du et al., 2003, 2004; Ihle et al., 2005;

70 Melchers et al., 2006). There are several known structural classes of tetraloops, three of which,

71 named according to consensus sequences, contain tetraloops of extreme stability: UNCG (where 
$72 \mathrm{~N}=$ any nucleotide), GNRA (where $\mathrm{R}=\mathrm{A} / \mathrm{G}$ ) and gCUUGc (Uhlenbeck, 1990; Cheong and

73 Cheong, 2010). Tetraloops of UNCG and GNRA classes are the most widely represented (Woese

74 et al., 1990; Cheong and Cheong, 2010; Bottaro and Lindorff-Larsen, 2017). Previously, it was

75 shown that only tetraloops of the UNCG structural class, but not tetraloops of GNRA or

76 gCUUGc structural classes, can support effective replication of the poliovirus genome (Prostova

77 et al., 2015). Moreover, the exact sequence of the apical region of poliovirus domain $\mathrm{d}$ was of

78 less importance for effective 3CD-oriL recognition than its spatial structure (Rieder et al., 2003;

79 Prostova et al., 2015). At the same time, structural disturbance in the apical region of oriL

80 domain d of poliovirus could be compensated by amino acid substitutions in the tripeptide 154-

81156 of the $3 \mathrm{C}$ protein (here and hereafter amino acid numeration corresponds to poliovirus $3 \mathrm{C}$

82 protein) (Andino et al., 1990; Prostova et al., 2015). In addition to triplet 154-156, the conserved

83 motif ${ }_{82} \mathrm{KFRDI}_{86}$ of the $3 \mathrm{C}$ protein also takes part in the oriL recognition (Andino et al., 1990,

84 1993; Hämmerle, Molla and Wimmer, 1992; Shih, Chen and Wu, 2004). To date a

85 comprehensive analysis of the diversity in domain $\mathrm{d}$ apical region and amino acid tripeptide

86 sequences in the Enterovirus genus has not been conducted.

87 The replication of an enterovirus is a low-fidelity process, generating, on average, one

88 mutation per genome (Sanjuán et al., 2010; Acevedo, Brodsky and Andino, 2013). The high

89 probability of mutation allows the virus to adapt to a constantly changing environment on the

90 one hand, but requires additional mechanisms to maintain genome stability on the other (Wagner 
91 and Stadler, 1999; Lauring, Frydman and Andino, 2013). The aim of the present study was to

92 understand co-evolution patterns of two interacting replication machinery elements in

93 enteroviruses, the apical domain $\mathrm{d}$ of oriL and the $3 \mathrm{C}$ protein.

\section{Materials and methods}

\section{Formation and filtration of sets of full genomes}

All available nucleotide sequences (as of February 2017) containing the Enterovirus

genus with length $8000>n>6800$ were extracted from the NCBI database. For every species, a

multiple sequence alignment was conducted using MAFFT version 7 with default settings (Katoh

and Standley, 2013). Sequences that contained more than $50 \mathrm{~N}$ characters in succession and sequences that were annotated as "Modified_Microbial_Nucleic_Acid", were removed from alignments. All sequences that differed from any other sequence in the dataset by less than $1 \%$ of

103 the nucleotide sequence were omitted in order to reduce the bias caused by over-represented

104 sequences.

For analysis of domain d sequence variety, the multiple sequence alignments were used.

107 The relevant region of multiple sequence alignment and the respective names of sequences were 
108 analysed in Microsoft Excel. To analyse correlation of the domain d loop and tripeptide of 3C

109 sequences the same alignments were translated in the protein $3 \mathrm{C}$ coding region. The resulting

110 amino acid sequences that corresponded to tripeptides 154-156 (poliovirus $3 \mathrm{C}$ numerations) were

111 analysed using Microsoft Excel. An amino acid frequency plot was created via the WebLogo

112 server using the set of filtered genomes for every species (Crooks et al., 2004). To do this, the

113 multiple sequence alignment of filtered genomes of every species was translated in the region

114 that codes protein 3C, while positions 71-89 and 147-160 were saved in separate MAS files,

115 which were then used to produce logos.

118 with subsequent manual editing (Gruber et al., 2008; Lorenz et al., 2011). Algorithm accounting

119 for minimum free energy and partition function was used.

\section{Results}

\section{Sample characteristics}

122 To evaluate the variability of the domain $\mathrm{d}$ loop sequence we retrieved all available

123 complete genome $(8000>n>6800$ nucleotides) enterovirus (EV) sequences that were present in

124 the NCBI database on February 2017. Representatives of Enterovirus A (1173 sequences in 
125 total), Enterovirus B (414), Enterovirus C (773), Enterovirus D (462), Enterovirus E (12),

126 Enterovirus F (13), Enterovirus G(15), Enterovirus H (3), Enterovirus J (7), Rhinovirus A (202),

127 Rhinovirus $B$ (76) and Rhinovirus $C$ (51) species were analysed. As expected, genomes of

128 epidemiologically significant viruses were the most represented in the database. For example,

$12966 \%$ of Enterovirus $A$ species genomes belonged to the EV71 type, the causative agent of hand,

130 foot and mouth disease (Solomon et al., 2010); most Enterovirus C species sequences (78\%)

131 belonged to poliovirus; and most Enterovirus D species sequences (98.7\%) represented EV68, an

132 aetiological agent of severe respiratory illness (Oermann et al., 2015). The number of genome

133 sequences of each species that contained the oriL region is shown in Table 1.

134 Sequences of apical regions in oriL domain $d$ and the amino acids involved in RNA

135 recognition in 3C protein were analysed. In genomes of Enterovirus $E$ and Enterovirus $F$ species

136 with two oriLs (Pilipenko, Blinov and Agol, 1990; Zell et al., 1999) sequences of both oriLs

137 were analysed (Table 1). To reduce the bias towards particular loop sequences present in a large

138 set of closely related genomes, which, for example, belonged to one outbreak, all sequences that

139 differed from any other sequence in the dataset by less than $1 \%$ of the nucleotide sequence were

140 omitted. After curation, the sizes of the largest data sets decreased dramatically, but the number

141 of unique loop sequences in every set did not change significantly (Table 1). Unique tetraloop

142 variants were lost for Enterovirus A (tetraloop UGUG), Enterovirus C (tetraloops CCCG, CAUG

143 and UGUG) and Enterovirus D (tetraloop UUGG). This indicates that, even among closely 
144 related genomes, the tetraloop sequence can vary. Indeed, in several outbreaks caused by EV71

145 or PV1, closely related genomes contained different apical domain d sequences (not shown). It

146 should be noted that the filtration of the dataset using a 95\% sequence identity threshold resulted

147 in a dramatic loss of unique tetraloop variants (107 genomes out of 1052 were left after filtration,

148 while 13 unique tetraloop variants out of total 17 variants were detected in the filtered set).

\section{Variability in the oriL domain d apical loop sequence}

150 The secondary structure of domain d was conserved in all species, except Enterovirus G,

151 in which an elongated domain d was observed (Figure 2) (Krumbholz et al., 2002).

152 The variety and occurrence of various loops in the apical region of domain $d$ in all

153 species of the Enterovirus genera were analysed in filtered sets of full genome sequences. Most

154 domain d apical loops were tetraloops (i.e., they consisted of four nucleotides) (Table 2).

155 However, triloops (3-nucleotide loop) could be found in genomes of Enterovirus $C$ and

Rhinovirus $A$ and $B$ species, whereas pentaloops (5-nucleotide loop) were detected in genomes of

157 Enterovirus E species (Table 2).

160 (tetraloops with UNCG class and gCUUGc class spatial structures belong to this consensus)

161 (Table 2). Consensus YNMG and consensus YNUG together corresponded to 24 unique

162 sequence variants. Interestingly, in our dataset of 2,842 full genomes, four tetraloops out of 24 
163 possible variants were never found in the domain $d$ apical region: UUAG, UCAG, CUAG and

164 CCAG (Table 2). Thus, dinucleotides UA and CA are likely to be avoided at positions 2 and 3 of

165 the tetraloop in enterovirus genomes.

166 In Enterovirus $A$ species, 17 out of 24 possible unique tetraloop sequences were

167 identified (Table 2, Table S2). Twelve unique loops of Enterovirus $A$ belonged to consensus

168 YNMG, while the other five belonged to consensus YNUG. The most abundant tetraloop in

169 Enterovirus $A$ genomes, in contrast to other species, was CUCG (Table 2, Table S1). This is

170 explained by the prevalence of this tetraloop in the EV71 C4 genotype (Table 2, Table S1).The

171 frequency of other tetraloop sequences varied significantly (Table 2, Table S2). One tetraloop

172 (UGUG) was lost upon filtration. Such sequences here and below were manually added to the

173 final data set to maintain diversity of loop sequences within the species, as well as provide

174 comprehensive information about sequences in apical domain $d$ in viable viruses (Table 2).

175 Interestingly, EV71 sequences contained 13 out of 17 tetraloop variants, which were detected in

176 the Enterovirus $A$ genus (Table 2). In other words, the diversity of tetraloops in one discrete

177 lineage in general resembles its diversity in the unification of different discrete lineages. In Enterovirus B genomes, 18 unique tetraloops out of 24 possible were found. Twelve

179 of these tetraloops belonged to consensus YNMG and six to consensus YNUG (Table 2, Table

180 S3). The most abundant tetraloops were CACG (98 genomes), UACG (51 genomes) and UGCG

181 (31 genomes), which were also present among the most abundant tetraloops of Enterovirus $A$ 
182 species.

183

In genomes of the Enterovirus $C$ species, nine unique tetraloops belonged to the YNMG

184

consensus and four to the YNUG consensus. Three unique tetraloops were lost upon filtration

185

and added to the final data set (CCCG, UGUG, CAUG) (Table 2, Table S4). Two genomes

186

annotated in the NCBI data base as Human coxsackievirus A21, strain Coe, (accession number

187

D00538) and Human coxsackievirus A21, strain BAN00-10467, (accession number EF015031)

188

contained triloops $\mathrm{CAG}$ and $\mathrm{CCG}$, respectively. The most abundant tetraloops in $E V$ - $C$ species

189

were UACG (106), CACG (101), UGCG (43) (Table 2, Table S1, Table S4), which corresponds

190

to the Sabin vaccine strains of poliovirus serotypes 2, 3 and 1, respectively. To evaluate bias

191

caused by the redundant number of vaccine strain sequences in the data set, we subtracted

192

genomes of vaccine/vaccine derived poliovirus strains from the analysed set. As a result,

tetraloops UACG, CACG and UGCG were still the most frequent variants (Table 2, Table S1).

194

Only 57 Enterovirus $D$ genomes out of 419 were left after $1 \%$ identity filtration. Fifty

genomes belonged to Human enterovirus 68, the aetiological agent of respiratory illness. All

196

genomes of this type contained loop UUCG in the domain d apical region. Other tetraloops were

UUUG (1), CUCG (2), CCCG (1), CUUG (2) and CACG (1) (Table 2, Table S5). One tetraloop

(UUGG) was lost upon filtration and manually added to the final data set.

199 Species Enterovirus $E$ and $F$ have two oriLs in the 5'NTR, generally with similar 
201 (Table S6). As such, we united sequences from the first and the second oriL of these viruses in 202 the heat map (Table 2). Domain d loops in 10 genomes of Enterovirus $F$ were tetraloops, while,

203 in 10 Enterovirus $E$ genomes, there were both tetraloops (first oriL) and pentaloops (first and

204 second oriL) (Table 2, Table S6). There were four diverse tetraloop sequences in oriLs of

205 Enterovirus $\mathrm{E}$ and $\mathrm{F}$ with no obvious preference between these species. These sequences were

206 GCUA, GUUA, GCCA, AUUA (Table 2, Table S6). Tetraloop AUUA was found once in the

207 first oriL domain d of EV-F (strain PS87/Belfast, accession number DQ092794) (Table 2, Table

208 S6). There were six diverse pentaloop sequences in domain $\mathrm{d}$ of Enterovirus E genomes -

209 GCUUA, GUUUA, GCCUA, GCGUA, GAUUA, GUCUA (Table 2, Table S6).

210 All domain d loops in genomes of Enterovirus G, H and J species were tetraloops; all

211 except one tetraloop variant belonged to consensus YNMG (Table 2, Table S7). One Enterovirus

212 G representative had a GUUA tetraloop sequence (strain LP 54, accession number AF363455),

213 similar to loops of Enterovirus E and F species (Table 2). This genome had only one oriL with

214 the same domain $d$ length as that of Enterovirus G genomes (Krumbholz et al., 2002).

215 All except one (isolate V38_URT-6.3m, accession number JF285329) of the full

216 genomes of Rhinovirus A species and all full genomes of Rhinovirus $C$ species had tetraloops in

217 the apical regions of domain d (Table 2). Tetraloops of these viruses in almost all cases belonged

218 to consensus YNMG, with one exception found in Rhinovirus C (tetraloop CUUC, isolate JAL-1,

219 accession number JX291115) (Table 2, Table S8). All loops in the apical region of Rhinovirus B 
220 domain d were triloops (Table 2, Table S8).

221 Thus, the secondary structure of domain $d$ was very similar among species of the

222 Enterovirus genus, with the exception of Enterovirus $G$ species (Figure 2). The apical region of

223 domain d has a high diversity of sequences; however, in species of Enterovirus $A, B, C, D, G, H$

224 and $J$ and Rhinovirus $A$ and $C$, it mostly corresponds to the same consensus, that is, YNHG

$225(\mathrm{Y}=\mathrm{C} / \mathrm{U}, \mathrm{N}=$ any, $\mathrm{H}=\mathrm{A} / \mathrm{C} / \mathrm{U})$.

226

\section{Variety of RNA-recognition tripeptide of 3C}

227

Two motifs of protein $3 \mathrm{C}$ are involved in RNA recognition and interact with oriL: the

228

conservative motif KFRDI (positions $82-86$ of poliovirus 3C) and the putative RNA-binding

229

tripeptide (positions 154-156 in poliovirus 3C) (Andino et al., 1990, 1993; Hämmerle, Hellen

230

and Wimmer, 1991; Shih, Chen and Wu, 2004). Substitutions in the putative RNA-binding

231 tripeptide are known to compensate for disturbance in the apical region of domain d, such that

232 the RNA-binding tripeptide is a putative candidate to co-evolve with the domain $\mathrm{d}$ loop (Andino

233 et al., 1990; Prostova et al., 2015). There are other amino acids that have been found to affect

234 oriL-3CD interaction, but tripeptide 154-156 (Enterovirus C 3C protein numbering here and

235 below) is the only one that is proven to compensate structural disturbance in the domain $\mathrm{d}$ apical

236 region (Andino et al., 1990, 1993). To evaluate the possible co-evolution between the domain $\mathrm{d}$

237 tetraloop and its putative interaction partners in protein $3 \mathrm{C}$, relevant sequences in the filtered full

238 genome data sets were analysed. 
240147 of the protease catalytic triad (Figure 3). Always in second position of the putative RNA-

241 binding tripeptide (position 155) was Gly.

242 No mutual dependence between loop sequences and tripeptide sequences was found

243 within enterovirus genomes of the same species. For example, Enterovirus $A$ genomes contained

24417 unique variants of the tetraloop sequence, whereas the predominant fraction of $3 \mathrm{C}$ sequences

245 (548 out of 564) contained the conservative tripeptide VGK at positions 154-156 (Figure 3,

246 Table S9). It is noteworthy that, this tripeptide was not found exclusively only in genomes of the

247 EV71 serotype, although genomes of this serotype prevailed in the data set. Other Enterovirus $A$

248 genomes contained tripeptides VGR (seven out of 564), TGK (four out of 564), IGK (three out

249 of 564), VGE (one out of 564) and SRK (one out of 564) (Figure 3, Table S9). Genomes with

250 tripeptides other than VGK contained no peculiarities of the domain d loop sequence (Table S9).

251 This observation confirms that the specific loop sequence is not likely to be the main subject for

252 recognition by the RNA-binding tripeptide. Similarly, all or almost all genomes of Enterovirus B

253 (242 out of 244), Enterovirus C (272 out of 274), Enterovirus D (all), Enterovirus $G$ (seven out

254 of 8), Enterovirus $H$ (a total of two: genomes - one with TGK, one with TGR), Enterovirus $J$

255 (all) and Rhinovirus B (36 out of 37) species contained tripeptide TGK at positions 154-156 of

256 the 3C protein (Figure 3, Table S7, S9, S10). Alternative tripeptides were TGR in two genomes

257 of Enterovirus B and one genome of Enterovirus $H$; IGK in one genome of Enterovirus $C$ 
258 species and in one genome of Rhinovirus B species; PGK in one genome of Enterovirus $C$

259 species; and MGK in one genome of Enterovirus $G$ species (Table S7, S9, S10).

260 Genomes of Enterovirus $E$ and $F$ species contained two oriLs with tetraloops in domain d

261 mostly of consensus GYYA or pentaloops of consensus GHBUA, where $\mathrm{H}=\mathrm{A} / \mathrm{C} / \mathrm{U}$ and

$262 \mathrm{~B}=\mathrm{U} / \mathrm{C} / \mathrm{G}$. All genomes contained tripeptide MGK at positions $154-156$ of protein $3 \mathrm{C}$ (Figure 3,

263 Table S7). Interestingly, a similar loop-tripeptide pair was found in one genome of Enterovirus $G$

264 species (strain LP 54, accession number AF363455). It contained tetraloop GUUA in domain d

265 of its single oriL and tripeptide MGK in 3C. Unlike this unique genome, other genomes of

266 Enterovirus $G$ species contained tetraloops of YNMG consensus and tripeptide TGK in the

267 protein $3 \mathrm{C}$.

Rhinovirus genomes contained tetraloops, mostly of consensus YNMG (Rhinovirus A and $C$ species) or triloops (Rhinovirus $A$ and $B$ ) (Table 2). Rhinovirus $A$ genomes with tetraloops in domain $\mathrm{d}$ contained tripeptides in $3 \mathrm{C}$ with positively charged amino acid before the tripeptide,

271 but not in its final position, as in case of genomes of Enterovirus $A-C$ species (Figure 3, Table

272 S11, S12). The sequence of tripeptides, which did not depend on the tetraloop sequence, was, in

273 descending order, IGQ (the most abundant, 65 genomes out of 119), IGL (20 genomes out of

274 119), IGS, VGS, IGN, VGQ, IGV and VGH (Table S11). In the case of the Rhinovirus A

275 genome with triloop UCU in domain d (isolate V38_URT-6.3m, accession number JF285329),

276 protein 3C contained tripeptide TGK without positively-charged amino acid before it (Table 
277 S11). All genomes of Rhinovirus $B$ species contained triloops in domain d, with all but one (with

278 IGK) containing tripeptide TGK in 3C. Genomes of Rhinovirus C contained tetraloops mostly of

279 consensus $\mathrm{YHCG}(\mathrm{H}=$ all but $\mathrm{G})$ and tripeptides in $3 \mathrm{C}$ without a positively charged amino acid at

280 the last position (TGN, VGN, TGH) or outside of the tripeptide (Table S12). One genome

281 contained tetraloop CUUC paired with most abundant tripeptide, that is, TGN (23 out of 37

282 genomes)(Table S12).

283 Thus, dependence between apical domain $\mathrm{d}$ sequences and tripeptides in protein $3 \mathrm{C}$

284 within a species was not detected (Figure 3). We can state that the tripeptide and motif KFRDI

285 are almost non-variable within a species compared to the domain d loop sequence, but there is a

286 specifically preferred tripeptide sequence for each species. Hence, tripeptide sequences are

287 species-specific, while the domain d loop sequences are almost universal among Enterovirus A,

$288 B, C$ and $D$ and Rhinovirus $A$ and $C$ species.

\section{Discussion}

Most of the domain $\mathrm{d}$ apical loops in enterovirus genomes were represented by tetraloops.

291 The most common variants of tetraloop sequences corresponded to consensuses $\mathrm{YNHG}(\mathrm{Y}=\mathrm{C} / \mathrm{U}$,

$292 \mathrm{~N}=$ any, $\mathrm{H}=\mathrm{A} / \mathrm{C} / \mathrm{U}$ ) (Table 2). Similar results were obtained in our previous experimental work,

293 where eight apical nucleotides of domain $d$ of the poliovirus genome were randomized and

294 viable variants were selected in vitro, with the majority of selected tetraloops belonging to 
295 consensus YNHG (Prostova et al., 2015). Some tetraloops of consensus YNHG were found in

296 genomes in the NCBI database, but not among the variants selected in vitro, namely tetraloops

297 CACG, CUCG, UAAG, UGAG, CAAG, CGAG, UGUG and CUUG (Prostova et al., 2015).

298 Tetraloops UGAG, UGUG and CUUG were reconstructed with a $\mathrm{U}^{* * * *} \mathrm{G}$ flanking base pair in

299 the context of the poliovirus genome strain Mahoney, which supported effective virus replication

300 (Prostova et al., 2015).

301 Conversely, tetraloops UUAG, UCAG and CCAG, found in domain d of selected in vitro

302 viable poliovirus variants, were able to support virus reproduction; however, they were not found

303 in naturally circulating viruses (Prostova et al., 2015). One tetraloop of the YNHG consensus

304 (CUAG) was neither found in genomes from the NCBI database $(n=2842)$, nor in the

305 randomized poliovirus genomes selected in vitro $(\mathrm{n}=62)$ (Table 2$)$. Thermodynamic stability is

306 unlikely to be the reason why this and other tetraloops were unrepresented as the melting

307 temperature of stem loops with avoided tetraloops is within range of the melting temperature of

308 YNHG tetraloops, which supported replication (Proctor et al., 2002). Moreover, tetraloops

309 UUAG and UCAG are common in rRNA (Woese et al., 1990). Sample insufficiency cannot be

310 excluded for both database and in vitro selected sets of genomes, but it is safe to conclude that

311 these tetraloop variants are at least extremely rare. In any case, the fact that the incidence of

312 these tetraloops is much less than for other tetraloops indicates that such variants are possibly

313 less fit. 
315 database and variants selected in vitro could be compiled into consensuses UNCG and CNCG

316 (Table 2, Table S1). At the same time, these tetraloops are most abundant in rRNA, and, with

317 certain closing base pairs, among the most thermodynamically stable tetraloops (Woese et al.,

318 1990; Proctor et al., 2002). Tetraloops of these consensuses and some other found tetraloops of

319 the YNHG consensus form a specific spatial structure of the UNCG structural class of stable

320 tetraloops (Cheong, Varani and Tinoco, 1990; Varani, Cheong and Tinoco, 1991; Du et al.,

$3212003,2004)$.

322

Another set of tetraloops, which correspond to GNYA consensus, was found both in

323 genomes of Enterovirus $E$ and $F$ and in genomes of viable polioviruses selected in vitro

324 (Prostova et al., 2015). Tetraloop GCUA was able to support the effective replication of

325 poliovirus and, together with tetraloop GUUA, is known to assume an UNCG fold (Ihle et al.,

326 2005; Melchers et al., 2006; Prostova et al., 2015). In sum, these data suggest that the spatial

327 structure, rather than the exact sequence, is the main subject for recognition by virus protein $3 \mathrm{C}$.

328 Structure-based recognition of tetraloops occurs in several known RNA-protein complexes. For

329 example, tetraloops with a GNRA class structure in the context of bacteriophages P22 and $\lambda$

330 genome transcription antitermination element boxB are specifically recognized by the

331 bacteriophage N-protein arginine-rich motif (ARM) (Cai et al., 1998; Legault et al., 1998;

332 Schärpf et al., 2000). Arginines and lysines of the ARM recognize the shape of the negatively 
333 charged phosphodiester backbone of the stem-loop and positions N-peptide for hydrophobic or

334 stacking interaction with a non-conserved nucleotide of the loop (Cai et al., 1998; Legault et al.,

335 1998; Schärpf et al., 2000; Thapar, Denmon and Nikonowicz, 2013). Another example of

336 structure-specific recognition is the complex of the double-stranded RNA-binding domain

337 (dsRBD) of RNase Rntlp and AGNN class tetraloop (Chanfreau, Buckle and Jacquier, 2000;

338 Lebars et al., 2001; Wu et al., 2001, 2004; Wang et al., 2011; Thapar, Denmon and Nikonowicz,

339 2013). Motif dsRBD recognizes the phosphodiester backbone at the 3' side of the tetraloop and

340 its non-conserved third and fourth nucleotides (Wu et al., 2004; Wang et al., 2011; Thapar,

341 Denmon and Nikonowicz, 2013).

342

The sequence to structure degeneracy (different RNA sequences are able to form similar

343

spatial structure) is the known phenomenon (Petrov, Zirbel and Leontis, 2013; Bottaro and

344 Lindorff-Larsen, 2017). Moreover, it is suggested to refrain from associating sequences with a

345 particular fold (D'Ascenzo et al., 2016, 2017). Together with the literature data our result let us

346 assume that sequence-structure degeneracy is an universal way in which RNA tetraloops are

347 used in nature (Lebars et al., 2001; Wu et al., 2004; Ihle et al., 2005; Petrov, Zirbel and Leontis,

348 2013; D’Ascenzo et al., 2016, 2017; Bottaro and Lindorff-Larsen, 2017).

349 It can be speculated that pentaloops in domain d of the Enterovirus $E$ genome and

350 triloops of domain $\mathrm{d}$ of rhinoviruses have the potential to comprise the same UNCG fold as some

351 YNHG and GNYA tetraloops. For HRV14 domain d, it was shown that its triloop resembles the 
352 structure of the first and last two nucleotides of UNCG structural class tetraloops (Headey et al.,

353 2007). There are pentaloops with four nucleotides that belong to consensus UNCG, GNRA or

354 gCUUGc, which are able to form spatial structures of corresponding structural classes with the

355 fifth bulged nucleotide (Cai et al., 1998; Schärpf et al., 2000; Theimer, Finger and Feigon, 2003;

356 Oberstrass et al., 2006; Liu et al., 2009). It is possible that four nucleotides of the pentaloops in

357 domain d of Enterovirus E species have a UNCG fold with one bulged nucleotide.

of natural and in vitro selected genomes. However, in an experiment such variants were found to evolve towards the YNHG or GNYA consensus (Prostova et al., 2015). Apparently, tetraloops may be beneficial under specific replication conditions. 
371 Enterovirus $A$ types (Lukashev et al., 2014); menawhile only one recombinant genome

372 (accession number HQ423143) was detected in our data set. Therefore, the variability of its

373 domain d loop sequence reflects changes that were only accumulated via mutations. The

374 diversity of the domain d loop sequence of EV-71 C4 viruses was far less prominent than among

375 Enterovirus $A$ genomes and represented only by five tetraloop sequence variants (Table 2). As

376 the most recent common ancestor of EV71 genotype C4 dates back about 20 years (McWilliam

377 Leitch et al., 2012), this diversity, although limited, has only emerged very recently. On the other

378 hand, the high sequence variability of the domain $d$ apical region in all enterovirus genomes was

379 possibly assisted by inter- and intra-species recombination events.

380 Interestingly, in contrast to the similar structure of domain $\mathrm{d}$ and the very similar

381 distribution of its apical sequences in genomes of different enterovirus species, its putative RNA-

382 recognition tripeptide of $3 \mathrm{C}$ is diverse (Figure 3). Most Enterovirus A genomes contain tripeptide

383 VGK in 3C, while there is a prevalence of the TGK tripeptide among genomes of Enterovirus B,

$384 C$ and $D$ species (Figure 3 ). Genomes of Rhinovirus $A$ and $C$ also contain common enterovirus

385 tetraloops in the domain $\mathrm{d}$ apical region, but, in 3C, unlike other species, they contain tripeptides

386 without positively charged amino acids (Figure 3, Table S11, Table S12). Positively charged

387 amino acids are often involved in the interaction with RNA, in particular, with phosphates of the

388 RNA backbone. As such, they are of importance to RNA-protein recognition (Jones et al., 2001;

389 Bahadur, Zacharias and Janin, 2008). In Rhinovirus A genomes, positively charged amino acid 
390 "jumped" from the last position of the tripeptide (position 156) to the position that precedes the

391 tripeptide (position 153) (Figure 3, shown by an arrow). The residue at position 153 starts and

392 the residue at position 156 ends the reverse turn between beta strands dII and eII of protein 3C

393 (Mosimann et al., 1997; Matthews et al., 1999; Cui et al., 2011). In a crystal structure of the

394 Rhinovirus A2 protein 3C, the side chain of Lys153 (preceding the tripeptide) is positioned in a

395 region similar to that of the side chain of Lys156 (in last position of the tripeptide) in the crystal

396 structure of Enterovirus 71 and Poliovirus 1 proteins 3C (Mosimann et al., 1997; Matthews et

397 al., 1999; Cui et al., 2011). Thus, Lys at position 153 of 3C has almost the same potential to

398 interact with the RNA-ligand as Lys at position 156 (Mosimann et al., 1997; Matthews et al.,

399 1999; Cui et al., 2011). Genomes of Rhinovirus $C$ species do not contain a positively charged

400 amino acid, either inside the tripeptide of the $3 \mathrm{C}$ protein, or in the neighbouring positions,

401 possibly indicating that tripeptide 154-156 in the protein 3C of Rhinovirus $C$ genome does not

402 interact directly with RNA. Thus, $3 \mathrm{C}$ is able to recognize domain $\mathrm{d}$ of the oriL with tripeptides of

403 a different sequence. In contrast to the domain $d$ structure and its apical sequence, the tripeptide

404 is species-specific. The diversity of the tripeptide, which is expected to recognize domain d, has

405 several compatible explanations. Residue 154 of the tripeptide possibly does not interact with

406 domain d directly. The tripeptide may be involved into a species-specific cooperative amino acid

407 network (amino acid “epistasis"). Moreover, different tripeptides could reflect slightly different

408 molecular mechanisms for domain d recognition. 
411 guaranteed to bind the same structured domain d. Genomes of the Rhinovirus $B$ contain triloops

412 in the apical region of domain d, which are paired with tripeptide TGK in $3 \mathrm{C}$, common for

413 genomes with tetraloops. In contrast, protein 3C of the Coxsackie virus B3 (Enterovirus B

414 species, containing tripeptide TGK) cannot recognize oriL sufficiently well when domain $\mathrm{d}$ is

415 capped with a triloop (Zell et al., 2002). This indicates that the sequence of the RNA-binding

416 tripeptide is probably not the exclusive participant in oriL-3C recognition. In other words,

417 different molecular mechanisms of oriL-3C recognition have evolved in every enterovirus

418 species independently. For example, it was shown for Rhinovirus 14 (Rhinovirus B species) that

419 protein $3 \mathrm{C}$ recognizes the stem region of domain d, rather than its apical loop (Leong et al., 1993). Another oriL-3C recognition mechanism is seemingly employed by Enterovirus $E$ and $F$

421 species, two oriLs of which play the same role as the single oriL in genomes of other

422 enteroviruses (Pilipenko, Blinov and Agol, 1990; Zell et al., 1999). The apical loop of their

423 domain $d$ is a tetra- or pentaloop with a sequence that differs from the loop consensuses of other

424 enteroviruses. The RNA-binding tripeptide in 3C is species-specific as well, and is always MGK

425 (Table S6). Interestingly, one genome of Enterovirus $G$ species had the same pair domain d loop:

426 tripeptide of 3C, i.e., GUUA MGK. Domain d of Enterovirus $G$ species is prolonged in

427 comparison to the length of domain $d$ in genomes of other species (Krumbholz et al., 2002) 
428 (Figure 2). Tripeptide MGK in the 3C of Enterovirus E, $F$ and $G$ possibly indicates another

429 molecular mechanism of oriL-3C recognition (Krumbholz et al., 2002). Therefore, we assume

430 that, though putative RNA-binding, the tripeptide, in most cases, possibly interacts with the

431 domain d apical region (since amino acid substitutions in it are known to compensate for

432 structural disturbance in domain d); however, this interaction is not the only one that determines

433 the evolution of oriL-3C interaction. Altogether, the data suggest that the independent evolution

434 of the putative RNA-binding tripeptide of $3 \mathrm{C}$ and domain $\mathrm{d}$ of oriL occurs.

435

\section{Conclusions}

and its protein ligand virus protease $3 \mathrm{C}$. RNA-binding motifs of $3 \mathrm{C}$ are species-specific, in

for Enterovirus $A, B, C$ and $D$ and Rhinovirus $A$ and $C$ species, whereas tripeptide sequence

almost universal diversity of tetraloop sequences among species, indicates the occurrence of the

444 sequence-based, recognition of domain d by virus protein $3 \mathrm{CD}$. These, together with the data

445 reported in the literature, let us assume that the sequence-structure degeneracy is a universal way 
446 RNA in which tetraloops are used in nature.

447

448 References

449 Acevedo, A., Brodsky, L. and Andino, R. (2013) 'Mutational and fitness landscapes of an RNA virus revealed through population sequencing.', Nature. Nature Publishing Group, 505(7485), pp. 686-690. doi: 10.1038/nature12861.

452 Andino, R., Rieckhof, G. E., Achacoso, P. L. and Baltimore, D. (1993) 'Poliovirus RNA synthesis utilizes an RNP complex formed around the 5' -end of viral RNA', EMBO Journal, 12(9), pp. 3587-3598. around the 5' End of Poliovirus', Cell, 63, pp. 369-380. protease (3Cpro) gene of poliovirus can suppress a mutation in the 5 ' noncoding region.', $J$ Virol, 64(2), pp. 607-612.

461 sites.', Nucleic Acids Res., 36(8), pp. 2705-2716. doi: 10.1093/nar/gkn102. 
466 Folds', Biophysical Journal, 113(2), pp. 257-267. doi: 10.1016/j.bpj.2017.06.011. complex.', Nature structural biology, 5(3), pp. 203-212. virology, 88(6), pp. 3192-3201. doi: 10.1128/JVI.02503-13.

of Sciences of the United States of America, 97(7), pp. 3142-3147. doi: 
484 (2011) 'Crystal structure of human enterovirus 71 3C protease.', Journal of molecular biology,

485 408(3), pp. 449-461. doi: 10.1016/j.jmb.2011.03.007.

486 D’Ascenzo, L., Leonarski, F., Vicens, Q. and Auffinger, P. (2016) ““Z-DNA like”

487 fragments in RNA: a recurring structural motif with implications for folding, RNA/protein 488 recognition and immune response.', Nucleic acids research, 44(12), pp. 5944-5956. doi: 10.1093/nar/gkw388. UNCG folds: U-turns versus Z-turns in RNA hairpin loops', RNA, 23(3), pp. 259-269. doi: 10.1261/rna.059097.116. 42(15), pp. 4373-4383. doi: 10.1021/bi027314e. 10.1021/bi048973p.

501 positive-stranded RNA virus', Genes \& Dev., 12, pp. 2293-2304. doi: 10.1101/gad.12.15.2293. 
503 and Evans, D. J. (2000) 'Identification of a cis-acting replication element within the poliovirus

504 coding region.', Journal of virology, 74(10), pp. 4590-4600. doi: 10.1128/JVI.74.10.4590-

$505 \quad 4600.2000$.

506 Gruber, A. R., Lorenz, R., Bernhart, S. H., Neuböck, R. and Hofacker, I. L. (2008) 'The

507 Vienna RNA websuite.', Nucleic acids research, 36(Web Server issue), pp. W70-4. doi:

$508 \quad 10.1093 / \mathrm{nar} / \mathrm{gkn} 188$.

509 Hämmerle, T., Hellen, C. U. and Wimmer, E. (1991) 'Site-directed mutagenesis of the

510 putative catalytic triad of poliovirus 3C proteinase.', The Journal of biological chemistry, 266(9),

511 pp. $5412-5416$.

512

Hämmerle, T., Molla, A. and Wimmer, E. (1992) 'Mutational analysis of the proposed

513 FG loop of poliovirus proteinase $3 \mathrm{C}$ identifies amino acids that are necessary for $3 \mathrm{CD}$ cleavage

514 and might be determinants of a function distinct from proteolytic activity.', $J$ Virol., 66(10), pp.

$515 \quad 6028-6034$.

516 Harris, K. S., Reddigari, S. R., Nicklin, M. J., Hämmerle, T. and Wimmer, E. (1992)

517 'Purification and characterization of poliovirus polypeptide $3 \mathrm{CD}$, a proteinase and a precursor

518 for RNA polymerase.', $J$ Virol., 66(12), pp. 7481-7489.

519 Headey, S. J., Huang, H., Claridge, J. K., Soares, G. A., Dutta, K., Schwalbe, M., Yang,

520 D. and Pascal, S. M. (2007) 'NMR structure of stem-loop D from human rhinovirus-14.', RNA,

521 13(3), pp. 351-360. doi: 10.1261/rna.313707. 
523 Ramachandran, R. and Görlach, M. (2005) 'A novel cGUUAg tetraloop structure with a

524 conserved yYNMGg-type backbone conformation from cloverleaf 1 of bovine enterovirus 1

525 RNA.', Nucleic Acids Res., 33(6), pp. 2003-2011. doi: 10.1093/nar/gki501.

'Protein - RNA interactions : a structural analysis', Biochemistry, 29(4), pp. 943-954. pp. 772-80. doi: 10.1093/molbev/mst010. and Zell, R. (2002) 'Sequencing of porcine enterovirus groups II and III reveals unique features of both virus groups.', Journal of virology, 76(11), pp. 5813-5821. 
542 'Solution structure of conserved AGNN tetraloops: insights into Rnt1p RNA processing.', The

543 EMBO journal, 20(24), pp. 7250-7258. doi: 10.1093/emboj/20.24.7250.

544 Legault, P., Li, J., Mogridge, J., Kay, L. E. and Greenblatt, J. (1998) 'NMR structure of

545 the bacteriophage lambda N peptide/boxB RNA complex: recognition of a GNRA fold by an

546 arginine-rich motif.', Cell, 93(2), pp. 289-299.

Leong, L. E. C., Walker, P. A., Porter, A. G., Protease, H. R.-, Leon, L. E. C., Walker, P.

A., Porter, A. G., Leong, L. E. C., Walker, P. A. and Porter, A. G. (1993) 'Human Rhinovirus-14

Protease 3C (3Cpro) Binds Specifically to the 5'-Noncoding Region of the Viral RNA', The

Journal of biological chemistry, 268(34), pp. 25735-25739.

Liu, P., Li, L., Keane, S. C., Yang, D., Leibowitz, J. L. and Giedroc, D. P. (2009) 'Mouse

552 hepatitis virus stem-loop 2 adopts a uYNMG(U)a-like tetraloop structure that is highly

553 functionally tolerant of base substitutions.', Journal of virology, 83(23), pp. 12084-12093. doi:

10.1128/JVI.00915-09.

555

Lorenz, R., Bernhart, S. H., Höner Zu Siederdissen, C., Tafer, H., Flamm, C., Stadler, P.

F. and Hofacker, I. L. (2011) 'ViennaRNA Package 2.0.', Algorithms for molecular biology,

6(26). doi: 10.1186/1748-7188-6-26.

558

Lukashev, A. N., Shumilina, E. Y., Belalov, I. S., Ivanova, O. E., Eremeeva, T. P., 
560 and evolutionary dynamics of the Human enterovirus A global gene pool.', The Journal of

561 general virology, 95(Pt 4), pp. 868-873. doi: 10.1099/vir.0.060004-0.

562 Matthews, D. a, Dragovich, P. S., Webber, S. E., Fuhrman, S. a, Patick, a K., Zalman, L.

563 S., Hendrickson, T. F., Love, R. a, Prins, T. J., Marakovits, J. T., Zhou, R., Tikhe, J., Ford, C. E.,

564 Meador, J. W., Ferre, R. a, Brown, E. L., Binford, S. L., Brothers, M. a, DeLisle, D. M. and

565 Worland, S. T. (1999) 'Structure-assisted design of mechanism-based irreversible inhibitors of

566 human rhinovirus $3 \mathrm{C}$ protease with potent antiviral activity against multiple rhinovirus

567 serotypes.', Proceedings of the National Academy of Sciences of the United States of America,

568 96(20), pp. 11000-11007.

569 McWilliam Leitch, E. C., Cabrerizo, M., Cardosa, J., Harvala, H., Ivanova, O. E., Koike,

570 S., Kroes, A. C. M., Lukashev, A., Perera, D., Roivainen, M., Susi, P., Trallero, G., Evans, D. J.

571 and Simmonds, P. (2012) 'The association of recombination events in the founding and

572 emergence of subgenogroup evolutionary lineages of human enterovirus 71.', Journal of

573 virology, 86(5), pp. 2676-2685. doi: 10.1128/JVI.06065-11.

574 Melchers, W. J. G., Zoll, J., Tessari, M., Bakhmutov, D. V, Gmyl, A. P., Agol, V. I. and

575 Heus, H. a (2006) 'A GCUA tetranucleotide loop found in the poliovirus oriL by in vivo SELEX

576 (un)expectedly forms a YNMG-like structure: Extending the YNMG family with GYYA.', RNA,

577 12(9), pp. 1671-1682. doi: 10.1261/rna.113106.

578 Mosimann, S. C., Cherney, M. M., Sia, S., Plotch, S. and James, M. N. (1997) 'Refined 
579 X-ray crystallographic structure of the poliovirus 3C gene product.', J Mol Biol, 273(5), pp.

580 1032-1047. doi: 10.1006/jmbi.1997.1306.

581 Muslin, C., Joffret, M.-L., Pelletier, I., Blondel, B. and Delpeyroux, F. (2015) 'Evolution

582 and Emergence of Enteroviruses through Intra- and Inter-species Recombination: Plasticity and

583 Phenotypic Impact of Modular Genetic Exchanges in the 5' Untranslated Region', PLOS

584 Pathogens, 11(11), p. e1005266. doi: 10.1371/journal.ppat.1005266.

585 Oberstrass, F. C., Lee, A., Stefl, R., Janis, M., Chanfreau, G. and Allain, F. H.-T. (2006)

586 'Shape-specific recognition in the structure of the Vts1p SAM domain with RNA.', Nature

587 structural \& molecular biology, 13(2), pp. 160-167. doi: 10.1038/nsmb1038.

588 Oermann, C. M., Schuster, J. E., Conners, G. P., Newland, J. G., Selvarangan, R. and

589 Jackson, M. A. (2015) 'Enterovirus D68. A focused review and clinical highlights from the 2014

590 U.S. Outbreak.', Annals of the American Thoracic Society, 12(5), pp. 775-781. doi:

591 10.1513/AnnalsATS.201412-592FR.

592 Palmenberg, A., Neubauer, D. and Skern, T. (2010) 'Genome organization and encoded

593 proteins.', in Ehrenfeld, E., Domingo, E., and Roos, R. P. (eds) The Picornaviruses. ASM Press, 594 pp. 3-17.

595 Petrov, A. I., Zirbel, C. L. and Leontis, N. B. (2013) 'Automated classification of RNA

596 3D motifs and the RNA 3D Motif Atlas', RNA, 19(10), pp. 1327-1340. doi:

597 10.1261/rna.039438.113. 
602 (2002) 'Isolation and characterization of a family of stable RNA tetraloops with the motif

603 YNMG that participate in tertiary interactions.', Biochemistry, 41(40), pp. 12062-12075. robustness and resilience of a replicative cis-element of RNA virus: promiscuity, limitations, relevance.', RNA biology, 12(12), pp. 1338-1354. doi: 10.1080/15476286.2015.1100794. 10.1099/vir.0.19013-0. mutation rates.', Journal of virology, 84(19), pp. 9733-9748. doi: 10.1128/JVI.00694-10. 
619 Biomedical Science, pp. 239-248. doi: 10.1159/000076036.

620

Solomon, T., Lewthwaite, P., Perera, D., Cardosa, M. J., McMinn, P. and Ooi, M. H.

621 (2010) 'Virology, epidemiology, pathogenesis, and control of enterovirus 71.', The Lancet.

622 Infectious diseases, 10(11), pp. 778-790. doi: 10.1016/S1473-3099(10)70194-8.

623 Thapar, R., Denmon, A. P. and Nikonowicz, E. P. (2013) 'Recognition modes of RNA

624 tetraloops and tetraloop-like motifs by RNA-binding proteins.', Wiley interdisciplinary reviews.

$625 R N A, 5(1)$, pp. 49-67. doi: 10.1002/wrna.1196.

626 Theimer, C. A., Finger, L. D. and Feigon, J. (2003) 'YNMG tetraloop formation by a

627 dyskeratosis congenita mutation in human telomerase RNA', $R N A$, 9, pp. 1446-1455. doi:

628 10.1261/rna.5152303.activity.

629 Thompson, A. A. and Peersen, O. B. (2004) 'Structural basis for proteolysis-dependent

630 activation of the poliovirus RNA-dependent RNA polymerase.', The EMBO journal, 23(17), pp.

631 3462-3471. doi: 10.1038/sj.emboj.7600357.

632 Trono, D., Andino, R. and Baltimore, D. (1988) 'An RNA sequence of hundreds of

633 nucleotides at the 5' end of poliovirus RNA is involved in allowing viral protein synthesis.',

634 Journal of virology, 62(7), pp. 2291-2299.

635 Uhlenbeck, O. C. (1990) 'Tetraloops and RNA folding', Nature. Nature Publishing 
636 Group, 346(6285), pp. 613-614. doi: 10.1038/346613a0.

637 Varani, G., Cheong, C. and Tinoco, I. (1991) 'Structure of an unusually stable RNA

638 hairpin.', Biochemistry, 30(13), pp. 3280-3289.

639 Vogt, D. A. and Andino, R. (2010) 'An RNA element at the 5'-end of the poliovirus

640 genome functions as a general promoter for RNA synthesis', PLoS pathogens, 6(6), p. e1000936.

641 doi: 10.1371/journal.ppat.1000936.

642

Wagner, A. and Stadler, P. F. (1999) 'Viral RNA and evolved mutational robustness.',

643 The Journal of experimental zoology, 285(2), pp. 119-127.

644

Wang, Z., Hartman, E., Roy, K., Chanfreau, G. and Feigon, J. (2011) ‘Structure of a yeast

645 RNase III dsRBD complex with a noncanonical RNA substrate provides new insights into

646 binding specificity of dsRBDs.', Structure, 19(7), pp. 999-1010. doi: 10.1016/j.str.2011.03.022.

647

Woese, C. R., Winker, S., Gutell, R. R., Winkers, S. and Gutell, R. R. (1990)

648

'Architecture of ribosomal RNA : Constraints on the sequence of "tetra-loops", Proceedings of

649

the National Academy of Sciences of the United States of America, 87(November), pp. 8467-

6508471.

651

Wu, H., Henras, A., Chanfreau, G. and Feigon, J. (2004) 'Structural basis for recognition

652

of the AGNN tetraloop RNA fold by the double-stranded RNA-binding domain of Rntlp RNase

653

III', Amino Acids, 101(22), pp. 8307-8312.

654

Wu, H., Yang, P. K., Butcher, S. E., Kang, S., Chanfreau, G. and Feigon, J. (2001) ‘A 
655 novel family of RNA tetraloop structure forms the recognition site for Saccharomyces cerevisiae

656 RNase III.', The EMBO journal, 20(24), pp. 7240-9. doi: 10.1093/emboj/20.24.7240.

657 Zell, R., Sidigi, K., Bucci, E., Stelzner, A. and Görlach, M. (2002) 'Determinants of the

658 recognition of enteroviral cloverleaf RNA by coxsackievirus B3 proteinase 3C.', RNA (New

659 York, N.Y.), 8(2), pp. 188-201. Available at:

$660 \mathrm{http}: / /$ www.pubmedcentral.nih.gov/articlerender.fcgi?artid=1370242\&tool=pmcentrez\&renderty

661 pe=abstract (Accessed: 19 December 2014).

662 Zell, R., Sidigi, K., Henke, A., Schmidt-Brauns, J., Hoey, E., Martin, S. and Stelzner, A.

663 (1999) 'Functional features of the bovine enterovirus 5'-non-translated region.', J Gen Virol, 80,

664 pp. 2299-2309.

665

666

667 
Figure 1

Schematic representation of poliovirus genome and detailed representation of secondary structure of poliovirus replicative element oriL.

Protein $3 \mathrm{C}$ and its RNA-binding motifs ${ }_{82} \mathrm{KFRDI}_{86}$ and ${ }_{154} \mathrm{TGK}_{156}$ are shown (here and below amino acid numeration corresponds to poliovirus protein 3C) (Prostova et al., 2015 with modifications).

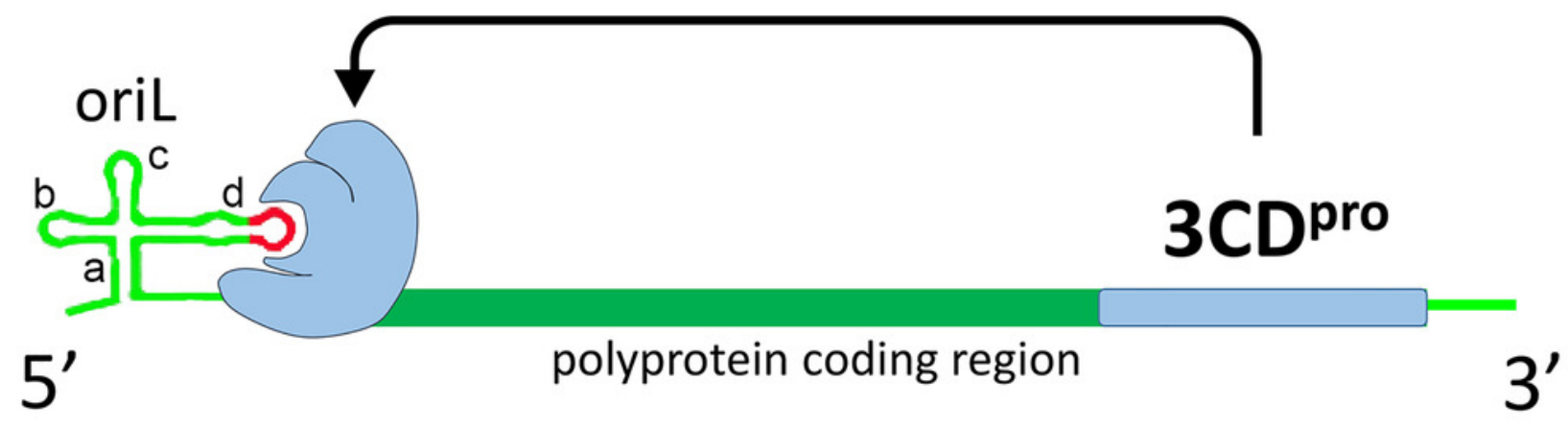


Figure 2

Secondary structure of oriL domain d of distinct enterovirus species.

For Enterovirus $E$ and $F$ domain $d$ of the first oriL is shown. Secondary structure of domain $d$ of Porcine enterovirus 9 strain UKG/410/73 was folded with use as reference Krumbholtz et al., 2002.

\section{$\underline{\text { Enterovirus }}$}

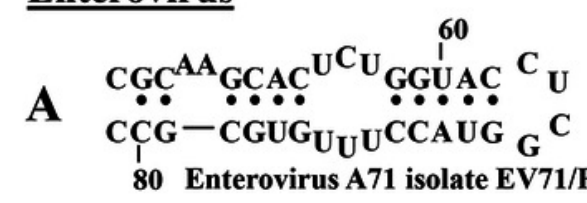

P123/2013/China

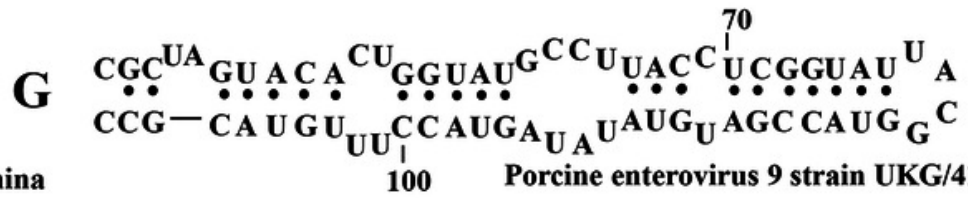

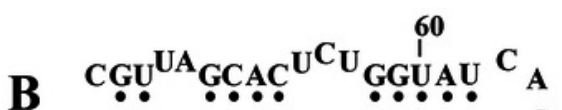
CCG - CGUG UUU CCAUG ${ }_{\mathbf{G}} \mathrm{C}$

80 Human echovirus 3, strain OC10-448

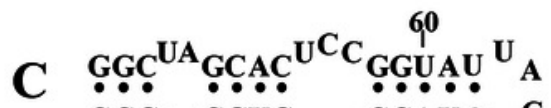
$\mathrm{CCG}_{\mathrm{S}}-\mathrm{CGUG}_{\mathrm{UU}_{U} \mathrm{CCAUG}} \mathrm{CH}_{\mathrm{G}} \mathrm{C}$

80 Human poliovirus 2 strain NIE0611432

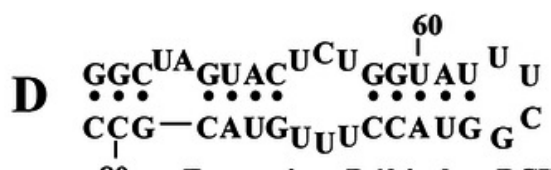

80 Enterovirus D68 isolate BCH895A

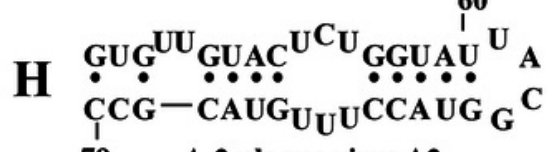

79 A-2 plaque virus A2

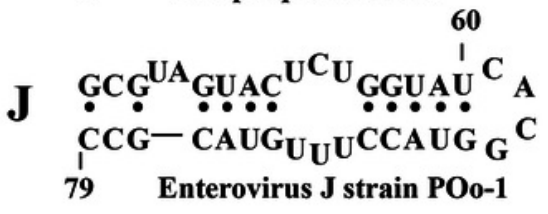

Rhinonirus $\quad 60$

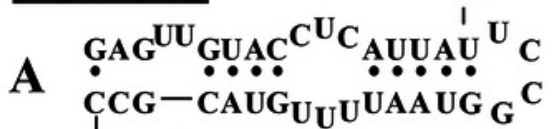

79 Human rhinovirus A21 isolate pumch13371/2013

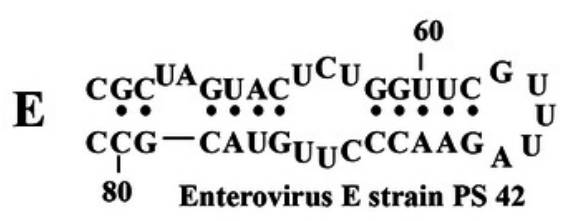

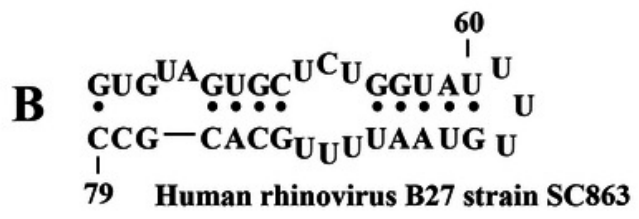

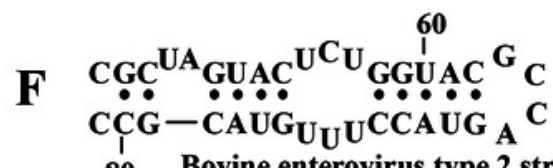

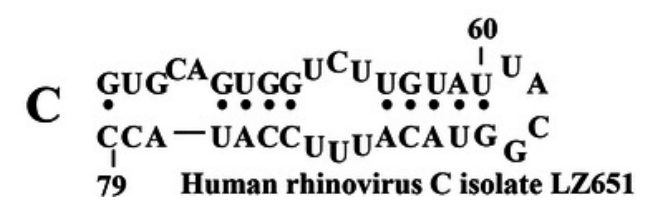




\section{Figure 3}

Distribituion of domain d loop sequence and amino acid motifs in the $3 \mathrm{C}$ protein.

A - Distribution of domain d loop sequences. The regions corresponding to tetraloop consensuses, triloops and pentaloops are shown. Number of genomes cut off at 15 for clear view of sequence distribution. B - The frequency plot of amino acid sequence of $3 \mathrm{C}$ in species of genus Enterovirus. The amino acid sequence logo was done with WebLogo server (Crooks et al., 2004). Arrows indicate amino acids of the proteolytic triade (Glu71 and Cys 147), the first and the last amino acids of motif ${ }_{82} \mathrm{KFRDI}_{86}$, the putative RNA-binding tripeptide 154-156 of $3 \mathrm{C}$ and Lys153 in the protein $3 \mathrm{C}$ of Rhinovirus $\mathrm{A}$. 
A

B
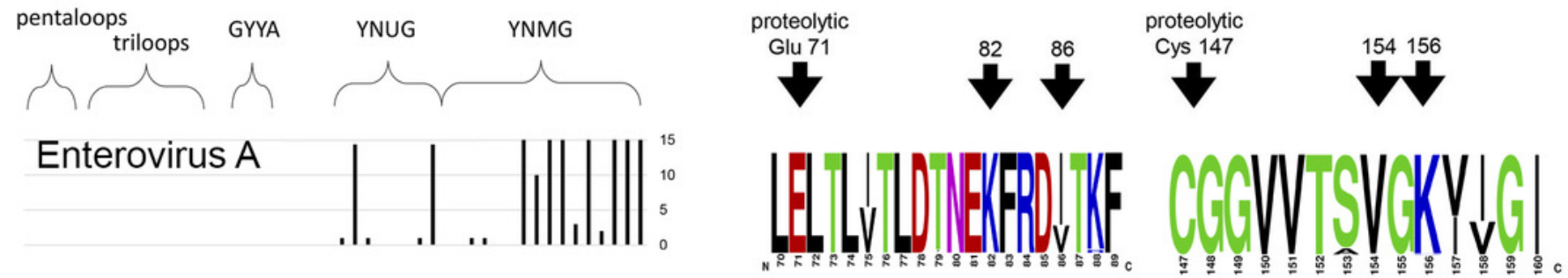

Enterovirus B

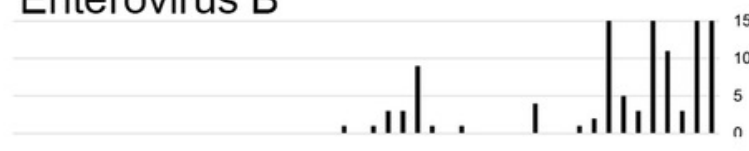

Enterovirus C

Enterovirus $\mathrm{F}$

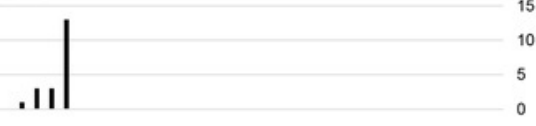

Rhinovirus A

$$
\left.\left.||_{1}\right|_{\mathbf{1}} ^{15}\right|_{0} ^{10}
$$

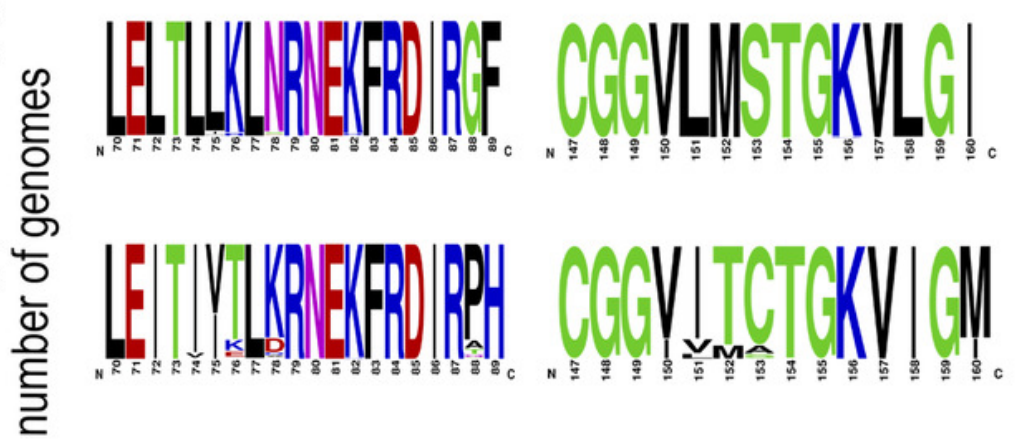

Rhinovirus B
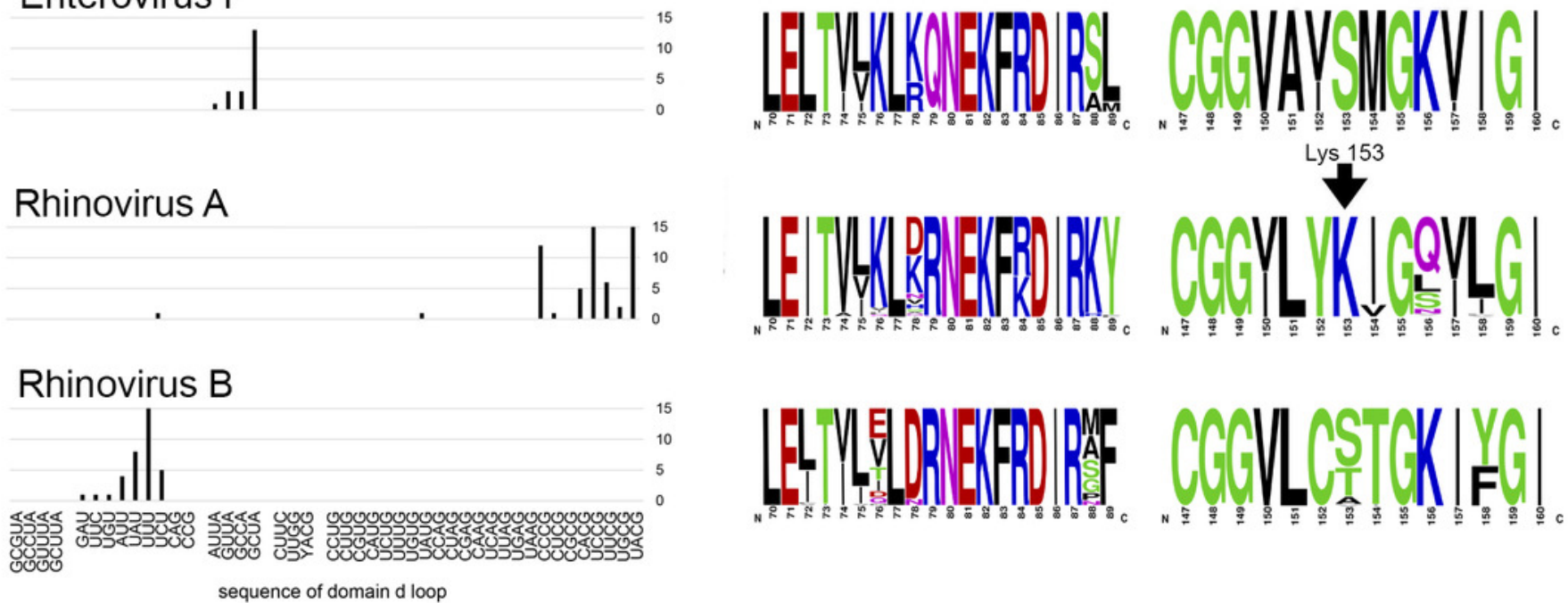


\section{Table $\mathbf{1}$ (on next page)}

Number of full genome sequences that contained oriL region and number of unique domain $\mathrm{d}$ sequences before and after filtration.

For Enterovirus $E$ and $F$ number of unique tetraloops is shown separately for first and the second oriL. 


\begin{tabular}{|c|c|c|c|c|c|c|}
\hline Species & $\begin{array}{l}\text { Number of } \\
\text { full genome } \\
\text { sequences }\end{array}$ & $\begin{array}{l}\text { Number of full genome } \\
\text { sequences after } 1 \% \\
\text { nucleic identity } \\
\text { filtration }\end{array}$ & \multicolumn{2}{|c|}{$\begin{array}{c}\text { Number of } \\
\text { unique } \\
\text { tetraloops before } \\
\text { filtration }\end{array}$} & \multicolumn{2}{|c|}{$\begin{array}{l}\text { Number of unique } \\
\text { tetraloops after } \\
\text { filtration }\end{array}$} \\
\hline Enterovirus A & 1052 & 564 & \multicolumn{2}{|c|}{17} & \multicolumn{2}{|c|}{16} \\
\hline Enterovirus B & 339 & 244 & \multicolumn{2}{|c|}{18} & \multicolumn{2}{|c|}{18} \\
\hline Enterovirus $\mathrm{C}$ & 747 & 274 & \multicolumn{2}{|c|}{15} & \multicolumn{2}{|c|}{12} \\
\hline Enterovirus D & 419 & 57 & \multicolumn{2}{|c|}{7} & \multicolumn{2}{|c|}{6} \\
\hline Enterovirus E & 12 & 10 & 6 & 5 & 6 & 5 \\
\hline Enterovirus $\mathrm{F}$ & 13 & 10 & 4 & 3 & 4 & 3 \\
\hline Enterovirus G & 10 & 8 & \multicolumn{2}{|c|}{6} & \multicolumn{2}{|c|}{6} \\
\hline Enterovirus $\mathrm{H}$ & 3 & 2 & \multicolumn{2}{|c|}{2} & \multicolumn{2}{|c|}{2} \\
\hline Enterovirus J & 8 & 5 & \multicolumn{2}{|c|}{3} & \multicolumn{2}{|c|}{3} \\
\hline Rhinovirus $\mathrm{A}$ & 151 & 118 & \multicolumn{2}{|c|}{8} & \multicolumn{2}{|c|}{8} \\
\hline Rhinovirus B & 50 & 37 & \multicolumn{2}{|c|}{7} & \multicolumn{2}{|c|}{7} \\
\hline Rhinovirus $\mathrm{C}$ & 38 & 37 & \multicolumn{2}{|c|}{6} & \multicolumn{2}{|c|}{6} \\
\hline
\end{tabular}

1 


\section{Table 2 (on next page)}

Occurrence of domain d apical sequences in filtered sets of full genomes of different enterovirus species.

Tetraloops CCCG, UGUG, CAUG and UUGG that were unique for species Enterovirus A, C and $D$ and were lost upon filtration, were added and are shown in blue. The gradient coloring from red to green represents abundancy heat map for the genomes with different domain $d$ sequence. 
1 Table 2 Occurrence of domain d apical sequences in filtered sets of full genomes of different

2 enterovirus species and serotypes. Tetraloops CCCG, UGUG, CAUG and UUGG that were unique

3 for species Enterovirus $A, B, C$ and $D$ and were lost upon filtration, were added to maintain the

4 diversity of loop sequence and are shown in blue. The gradient coloring from red to green

5 represents abundance heat map for the genomes with different domain $\mathrm{d}$ sequence.

6

\begin{tabular}{|c|c|c|c|c|c|c|c|c|c|c|c|c|c|c|c|c|c|}
\hline \multirow{3}{*}{$\begin{array}{l}\text { Loop } \\
\text { sequence }\end{array}$} & \multicolumn{14}{|c|}{ Enterovirus } & \multicolumn{3}{|c|}{ Rhinovirus } \\
\hline & \multicolumn{4}{|c|}{ A } & \multirow[b]{2}{*}{ B } & \multicolumn{3}{|c|}{ C } & \multirow[b]{2}{*}{ D } & \multirow[b]{2}{*}{$\mathrm{E}$} & \multirow[b]{2}{*}{$\mathrm{F}$} & \multirow[b]{2}{*}{ G } & \multirow[b]{2}{*}{$\mathrm{H}$} & \multirow[b]{2}{*}{$\mathrm{J}$} & \multirow[b]{2}{*}{ A } & \multirow[b]{2}{*}{ B } & \multirow[b]{2}{*}{ C } \\
\hline & all & EV71 & $\begin{array}{l}\text { EV71 C4 } \\
\text { genotype }\end{array}$ & $\begin{array}{l}\text { non } \\
\text { EV71 }\end{array}$ & & all & PV & $\begin{array}{l}\text { non } \\
\text { PV }\end{array}$ & & & & & & & & & \\
\hline \multicolumn{18}{|c|}{ Triloops } \\
\hline CCG & & & & & & 1 & & 1 & & & & & & & & & \\
\hline CAG & & & & & & 1 & & 1 & & & & & & & & & \\
\hline UCU & & & & & & & & & & & & & & & 1 & 5 & \\
\hline UUU & & & & & & & & & & & & & & & & 17 & \\
\hline UAU & & & & & & & & & & & & & & & & 8 & \\
\hline AUU & & & & & & & & & & & & & & & & 4 & \\
\hline UGU & & & & & & & & & & & & & & & & 1 & \\
\hline UUC & & & & & & & & & & & & & & & & 1 & \\
\hline GAU & & & & & & & & & & & & & & & & 1 & \\
\hline \multicolumn{18}{|c|}{ YNMG Tetraloops } \\
\hline UACG & 85 & 28 & & 57 & 51 & 106 & 64 & 42 & & & & 3 & 1 & 2 & 38 & & 15 \\
\hline UGCG & 114 & 2 & & 112 & 31 & 43 & 31 & 12 & & & & 1 & 1 & & 2 & & \\
\hline UUCG & 16 & 16 & 14 & & 3 & & & & 50 & & & & & & 6 & & 6 \\
\hline UCCG & 2 & & & 2 & 11 & 1 & 1 & & & & & & & & 53 & & 10 \\
\hline CACG & 48 & 28 & & 20 & 98 & 101 & 54 & 47 & 1 & & & 1 & & 2 & 5 & & \\
\hline CGCG & 3 & 2 & & 1 & 3 & 13 & 6 & 7 & & & & 1 & & & . & & \\
\hline CUCG & 132 & 127 & 126 & 5 & 5 & 2 & & 2 & 2 & & & & & & 1 & & 3 \\
\hline CCCG & 40 & 39 & 28 & 1 & 16 & 1 & & 1 & 1 & & & & & & 12 & & 2 \\
\hline UAAG & 10 & 10 & & & 2 & & & & & & & & & & & & \\
\hline UGAG & 22 & 22 & & & 1 & & & & & & & & & & & & \\
\hline \multirow{2}{*}{\multicolumn{18}{|c|}{$\begin{array}{l}\text { UUAG } \\
\text { UCAG }\end{array}$}} \\
\hline & & & & & & & & & & & & & & & & & \\
\hline CAAG & 1 & 1 & & & 4 & 1 & & 1 & & & & & & 1 & & & \\
\hline CGAG & 1 & & & 1 & & 2 & & 2 & & & & & & & & & \\
\hline CUAG & & & & & & & & & & & & & & & & & \\
\hline CCAG & & & & & & & & & & & & & & & & & \\
\hline YACG & & & & & 1 & & & & & & & & & & & & \\
\hline & & & & & & YNU & Tetra & ops & & & & & & & & & \\
\hline UAUG & 54 & 1 & & 53 & & & & & & & & & & & 1 & & \\
\hline UGUG & 1 & & & 1 & 1 & 1 & & 1 & & & & & & & & & \\
\hline UUUG & & & & & & & & & 1 & & & & & & & & \\
\hline UCUG & & & & & 1 & & & & & & & & & & & & \\
\hline CAUG & & & & & 9 & 1 & & 1 & & & & & & & & & \\
\hline CGUG & 1 & & & 1 & 3 & 2 & & 2 & & & & & & & & & \\
\hline CUUG & 34 & 34 & 35 & & 3 & & & & 2 & & & & & & & & \\
\hline CCUG & 1 & 1 & 1 & & 1 & 1 & & 1 & & & & & & & & & \\
\hline & & & & & & GYY & Tetra & ops & & & & & & & & & \\
\hline GCUA & & & & & & & & & & 2 & 13 & & & & & & \\
\hline GCCA & & & & & & & & & & & 3 & & & & & & \\
\hline GUUA & & & & & & & & & & 2 & 3 & 1 & & & & & \\
\hline & & & & & & Othe & etra & ops & & & & & & & & & \\
\hline
\end{tabular}




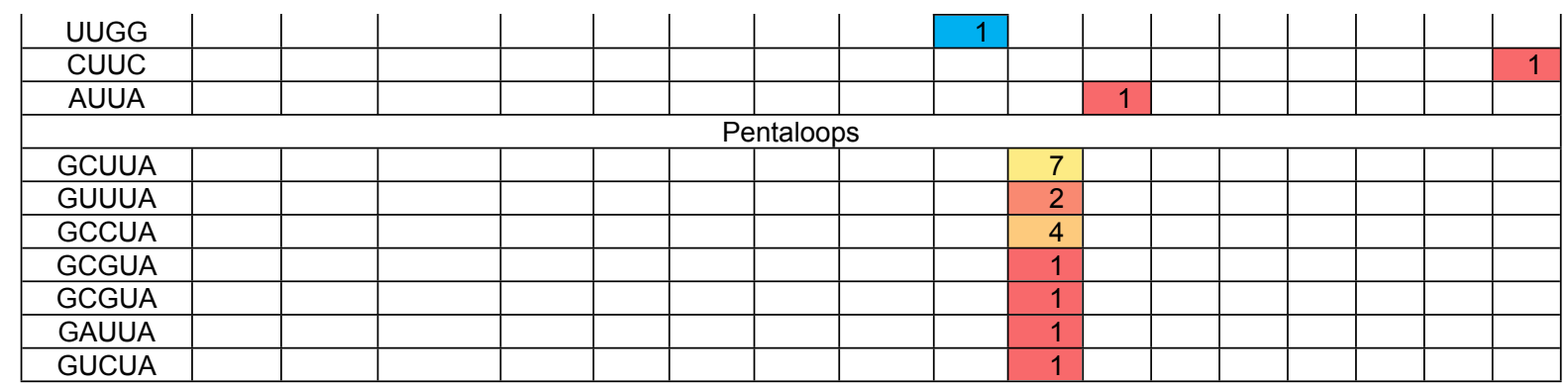

